\title{
Substituição de prótese inferior tipo protocolo para overdenture em paciente geriátrico: relato de caso
}

\author{
Replacement of a lower implant-supported prosthesis for overdenture in a geriatric patient: a case
} report

\author{
Sustitución de una prótesis inferior implantosoportada por sobredentadura en un paciente \\ geriátrico: reporte de caso
}

\author{
Camila de Carvalho Almança Lopes \\ ORCID: https://orcid.org/0000-0001-9313-9818 \\ Universidade Federal de Uberlândia, Brasil \\ E-mail: milalopes_81@ hotmail.com \\ Paula Lourenço Sousa \\ ORCID: https://orcid.org/0000-0002-6234-0719 \\ Universidade Federal de Uberlândia, Brasil \\ E-mail: paulinha.sousa@hotmail.com \\ Paulo Cézar Simamoto Júnior \\ ORCID: https://orcid.org/0000-0001-6087-9721 \\ Universidade Federal de Uberlândia, Brasil \\ E-mail: psimamoto@gmail.com \\ Erick Rene Cerda-Rizo \\ ORCID: https://orcid.org/0000-0003-4316-1122 \\ Universidad Americana, Nicaragua \\ E-mail: erickcerda8@gmail.com \\ Brenda Lisseth Pineda Mancia \\ ORCID: https://orcid.org/0000-0002-9243-381X \\ Universidade de Brasília, Brasil \\ E-mail: drapinedamancia@gmail.com \\ Morgana Guilherme de Castro Silverio \\ ORCID: https://orcid.org/0000-0002-0352-7946 \\ Universidade Federal de Uberlândia, Brasil \\ E-mail: morgana.guilherme@ufu.br
}

\begin{abstract}
Resumo
O endentulismo é um problema de saúde no Brasil, pois afeta o paciente gerando problemas psíquicos, sociais e funcionais. Os tratamentos com próteses sobre implantes devem ser muito bem planejados e executados, para ter uma adequada manutenção e longevidade. Desse modo o presente trabalho relata caso clínico de substituição de uma prótese do tipo protocolo de Branemark inferior por uma overdenture, considerando os princípios biopsicossociais do paciente. Paciente do sexo feminino, 92 anos de idade, compareceu projeto de extensão Overdenture para desdentados totais na geriatria na Escola Técnica de Saúde (ESTES-UFU), queixando-se de halitose, dificuldade de higienização do protocolo e desconforto geral com as próteses. O exame clínico intraoral revelou a presença de uma prótese mucosoportada superior e uma prótese fixa do tipo protocolo inferior, a qual apresentava uma inadequada estabilidade oclusal, alto grau de desgaste dos dentes de resina e presença de biofilme, revelando uma inabilidade da paciente de realizar a correta higienização, visto a perda de coordenação motora dada pela idade da paciente. O plano de tratamento foi definido pela adequação do meio bucal, orientação de escovação e posteriormente a confecção de duas próteses novas. Foram confeccionadas uma prótese convencional superior e uma prótese overdenture inferior sobre pilares Equator, aproveitando os 4 implantes em adequada posição. Após a instalação das próteses, foi possível diagnosticar um maior conforto mastigatório e uma melhora na higienização. Assim, conseguiu-se promover qualidade e longevidade no tratamento de acordo com a necessidade e idade da paciente, devolvendo a saúde oral e a autoestima.
\end{abstract}

Palavras-chave: Prótese dentária; Reabilitação bucal; Qualidade de vida.

\section{Abstract}

Endentulism is a health problem in Brazil, as it affects the patient generating psychological, social and functional problems. Treatments with implant supported prostheses must be very well planned and executed, to have an adequate maintenance and longevity. Thus, the present study reports a clinical case of replacement of a lower implant- 
supported prothesis in accordance with the traditional Branemark protocol by an overdenture, considering the patient's biopsychosocial principles. A 92-year-old female patient attended an Overdenture extension project for total toothless in geriatrics at the Escola Técnica de Saúde (ESTES-UFU), complaining of halitosis, difficulty in cleaning the implant supported prostheses and general discomfort with the prostheses. The intraoral clinical examination revealed the presence of a superior mucosal prosthesis and a implant supported prostheses in the mandibule, which presented inadequate occlusal stability, high degree of wear of the resin teeth and the presence of biofilm, revealing an inability of the patient to perform the correct cleaning, given the loss of motor coordination by the patient's age. The treatment plan was defined by the adequacy of the oral environment, brushing orientation and later the manufacture of two new prostheses. A conventional upper prosthesis and a lower overdenture prosthesis over Equator abutments were made, taking advantage of the 4 implants in an adequate position. After installing the prostheses, it was possible to diagnose greater chewing comfort and an improvement in hygiene. Thus, it was possible to promote quality and longevity in the treatment according to the patient's need and age, restoring oral health and self-esteem.

Keywords: Dental prosthesis; Mouth rehabilitation; Quality of life.

\section{Resumen}

El endentulismo es un problema de salud en Brasil, ya que afecta al paciente, generando problemas psicológicos, sociales y funcionales. Los tratamientos con prótesis sobre implantes deben estar muy bien planificados y ejecutados, para tener un adecuado mantenimiento y longevidad. Así, el presente trabajo reporta un caso clínico de sustitución de una prótesis del tipo de protocolo Branemark inferior por una sobredentadura, considerando los principios biopsicosociales del paciente. Paciente femenina de 92 años que acudió a un proyecto de extensión de Sobredentadura para desdentado total en geriatría en la Escuela Técnica de Salud (ESTES-UFU), que consultaba por halitosis, dificultad en la limpieza del protocolo y malestar generalizado con las prótesis. El examen clínico intraoral reveló la presencia de una prótesis total mucososoportada superior y una prótesis fija del tipo protocolo inferior, las cuales presentaban una estabilidad oclusal inadecuada, alto grado de desgaste de los dientes de resina y presencia de biofilm, revelando la incapacidad del paciente para realizar el procedimiento. Higiene incorrecta, debido a la pérdida de coordinación motora por la edad del paciente. El plan de tratamiento se definió por la adecuación del medio bucal, la orientación del cepillado y posteriormente la fabricación de dos nuevas prótesis. Se realizó una prótesis superior convencional y una sobredentadura inferior sobre pilares Equator, aprovechando los 4 implantes en una posición adecuada. Posterior a la instalación de las prótesis, se pudo diagnosticar una mayor comodidad masticatoria y una mejora en la higiene. Así, se logró promover la calidad y longevidad en el tratamiento de acuerdo a la necesidad y edad del paciente, restableciendo la salud bucal y la autoestima.

Palabras clave: Prótesis dental; Rehabilitación bucal; Calidad de vida.

\section{Introdução}

O edentulismo é uma condição que representa um problema comum de saúde da população idosa. Atualmente, países como o Brasil ainda apresentam altas taxas de prevalência de edentulismo (da Silva, de Oliveira, \& Leles 2015), gerando consequências como limitações funcionais, estéticas e psicossociais que afligem negativamente a qualidade de vida dos pacientes (de Souza et al., 2016; Sánchez-Siles et al., 2017; Kutkut et al., 2018).

Diferentes tratamentos são propostos com o objetivo de melhorar a qualidade de vida dos pacientes desdentados totais, como a prótese total convencional, a prótese removível sobre implantes e as próteses fixas sobre implantes (metaloplástica, metalocerâmica ou cerâmica pura) (Kern, Kern, Wolfart, \& Heussen, 2016; Gonçalves et al., 2020). Entretanto, particularmente quando uma prótese total convencional é instalada na mandíbula, ela pode ficar instável e ser desconfortável para o paciente, dificultando a execução de tarefas essenciais como a mastigação e a fala. Pacientes que fazem uso de próteses totais convencionais podem sofrer de disfunções crônicas, dor, baixa autoestima e pobre qualidade de vida (Rivaldo, Montagner, Nary, da Fontoura Frasca, \& Branemark, 2012).

Diante disso e pautando-se na necessidade de buscar métodos mais eficientes, o tratamento com implantes orais com o passar do tempo foi aperfeiçoando-se. As primeiras próteses sobre implante foram realizadas em pacientes desdentados inferiores, sendo confeccionada em duas etapas. O protocolo original de Branemark utilizava 5 a 6 implantes interforaminais, com um tempo de osseointegração de 3 a 6 meses, para posterior confecção das próteses (Adell, Eriksson, Lekholm, Brånemark, \& Jemt, 1990). Entretanto, atualmente a reabilitação pode ocorrer em até uma única fase, procedimento cirúrgico e carga imediata, associado a redução do número de implantes de apoio (Rivaldo et al., 2012; Freitas et al., 2021). 
As próteses sobre implantes garantem melhor atividade neuromuscular, portanto, auxiliam no processo de mastigação (van der Bilt, van Kampen, \& Cune, 2006; Fueki, Kimoto, Ogawa, \& Garrett, 2007; Geckili et al., 2012) devido a sua estabilidade e capacidade de retenção (Naert, Quirynen, Hooghe, \& van Steenberghe, 1994; Cordioli, Majzoub, \& Castagna, 1997; de Souza et al., 2016; Alfadda \& Attard, 2017). Entretanto, com o passar do tempo, as próteses fixas sobre implantes podem apresentar algumas limitações para o paciente, tendo em vista a diminuição de sua coordenação motora com o avançar da idade e, consequentemente, a dificuldade em promover a higienização (Delwel et al., 2017). Portanto, os tratamentos com próteses sobre implantes devem ser bem planejados e executados para obter longevidade cumulada com facilidade de sua manutenção (Cooper, Limmer, \& Gates, 2012; Zancope, Simamoto Junior, Davi, Prado, \& Neves, 2014; Kutkut et al., 2018).

Com o aumento da expectativa de vida, as overdentures sobre implantes (próteses totais mucossuportadas e implantoretidas) apresentam vantagens para o paciente idoso, favorecendo a higienização adequada, qualidade e longevidade do tratamento de acordo com suas necessidades e limitações, reabilitando a saúde bucal e a autoestima do paciente (Elsyad \& Shawky, 2017). Além disso, o grau de estabilidade das próteses overdenture melhoram as funções orais em relação as próteses convencionais, favorecendo uma retenção adequada, mastigação satisfatória e melhoria na fonética, aumentando assim a qualidade de vida do paciente (Elsyad \& Shawky, 2017).

Para obtenção de bons resultados, é necessário entender os diferentes sistemas de retenção disponíveis, dentre os principais sistemas, temos: o sistema barra-clipe, os sistemas independentes e os sistemas magnéticos (Chung, Chung, Cagna, \& Cronin, 2004). O sistema barra-clipe funciona no sentido de promover um encaixe de um clipe a uma barra que se une a dois ou mais implantes. Já os sistemas independentes apresentam-se como um mecanismo macho/fêmea, no qual o componente macho é fixado ao implante e o componente fêmea fixado na prótese. Por fim, os sistemas magnéticos constituem-se de um imã e um componente magnético, sendo o imã fixado na prótese e o componente magnético parafusado ao implante (Elsyad \& Khairallah, 2017; Elsyad \& Shawky, 2017; Scherer, McGlumphy, Seghi, \& Campagni, 2014). Sendo que a escolha do correto sistema de retenção corrobora para o sucesso do tratamento (Bhargava, Sehgal, Gupta, \& Mehra, 2016).

Diante do exposto, este artigo tem como objetivo descrever o passo a passo acerca da substituição de prótese total convencional superior e protocolo metaloplástica inferior insatisfatória por prótese total convencional superior e overdenture inferior sobre sistemas independentes, considerando os princípios biomecânicos e biopsicossocial da paciente.

\section{Metodologia}

O artigo trata-se de um relato de caso clínico descritivo e qualitativo. Pereira et al., (2018) referem-se a esse tipo de manuscrito como uma descrição e análise o mais detalhada possível de algum caso que apresente alguma particularidade que o torna especial. A paciente procurou a Faculdade de Odontologia da Universidade Federal de Uberlândia para tratamento odontológico (Uberlândia/MG) e após concordar com o plano de tratamento proposto, a paciente assinou termo de consentimento livre e esclarecido.

\section{Relato de Caso}

Paciente do sexo feminino, 92 anos, buscou atendimento no projeto de extensão Overdenture para desdentados totais na geriatria na Escola Técnica de Saúde (ESTES-UFU), com queixa de forte halitose, dificuldade em higienizar a prótese inferior, desconforto na mucosa inferior, dificuldade de se alimentar e consequentemente baixa autoestima pelo mal-estar gerado com as próteses. Ao exame clínico, observou-se a presença de uma prótese total mucossuportada na maxila e uma prótese fixa metaloplástica implantossuportada (prótese tipo protocolo) retida por quatro implantes na mandíbula (Figuras $1 \mathrm{e}$ 2). Elas apresentavam baixa estabilidade oclusal, alto desgaste dos dentes, presença de manchas (Figura 2) e, especificamente na protocolo inferior, verificou-se uma grande quantidade de biofilme (Figura 3), demonstrando a dificuldade da paciente para 
higienizá-la adequadamente devido à perda da coordenação motora gerada pela própria idade e do grande volume de resina da base do protocolo.

Figura1: Aspecto extra oral para avaliação inicial e planejamento do caso.

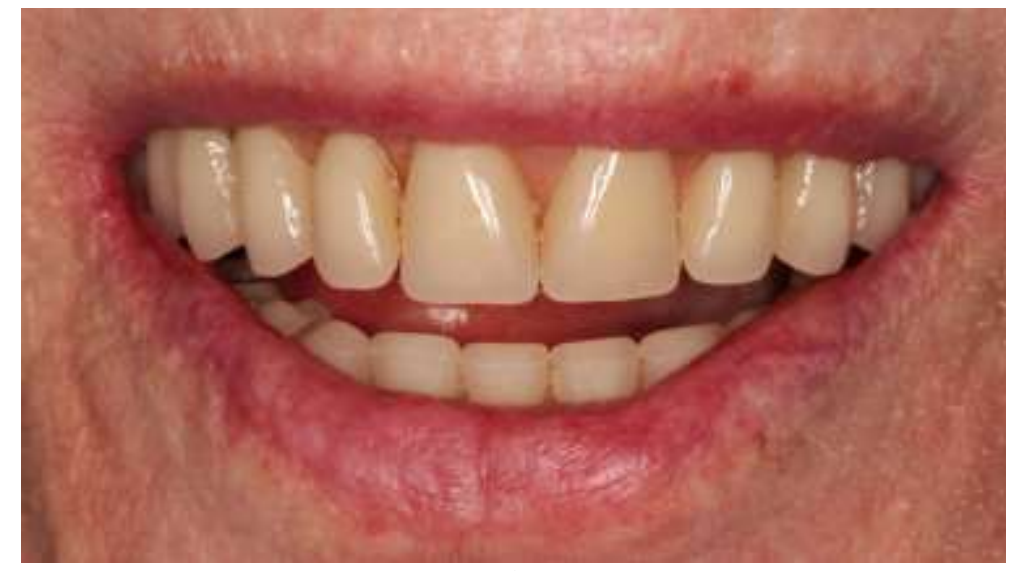

Fonte: Autores.

Figura 2: Ao exame intra-oral, observou-se a presença de uma prótese total superior e de protocolo inferior.

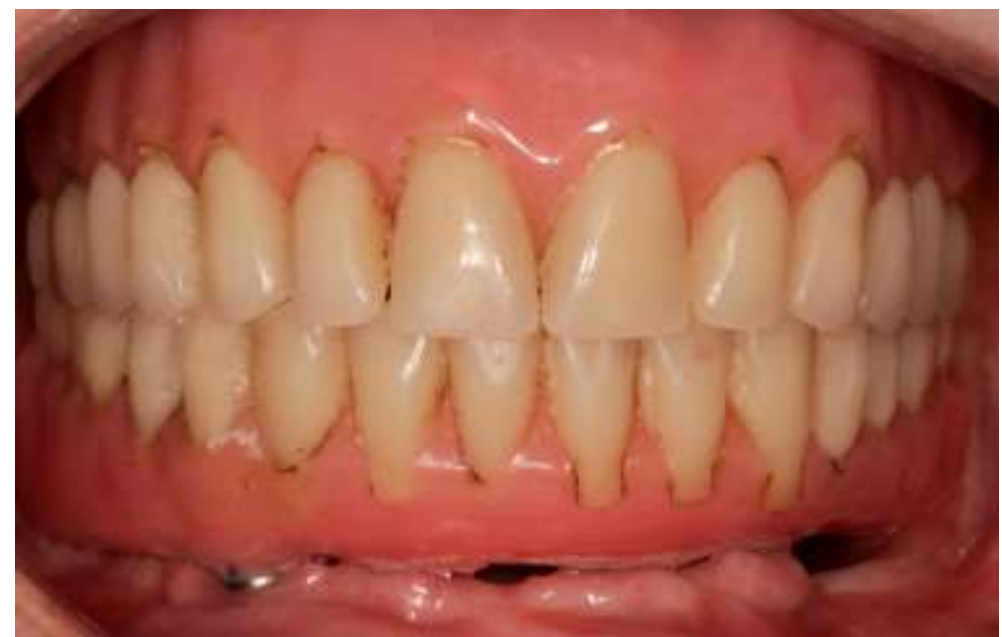

Fonte: Autores. 
Figura 3: Acúmulo de placa na base da protocolo inferior.

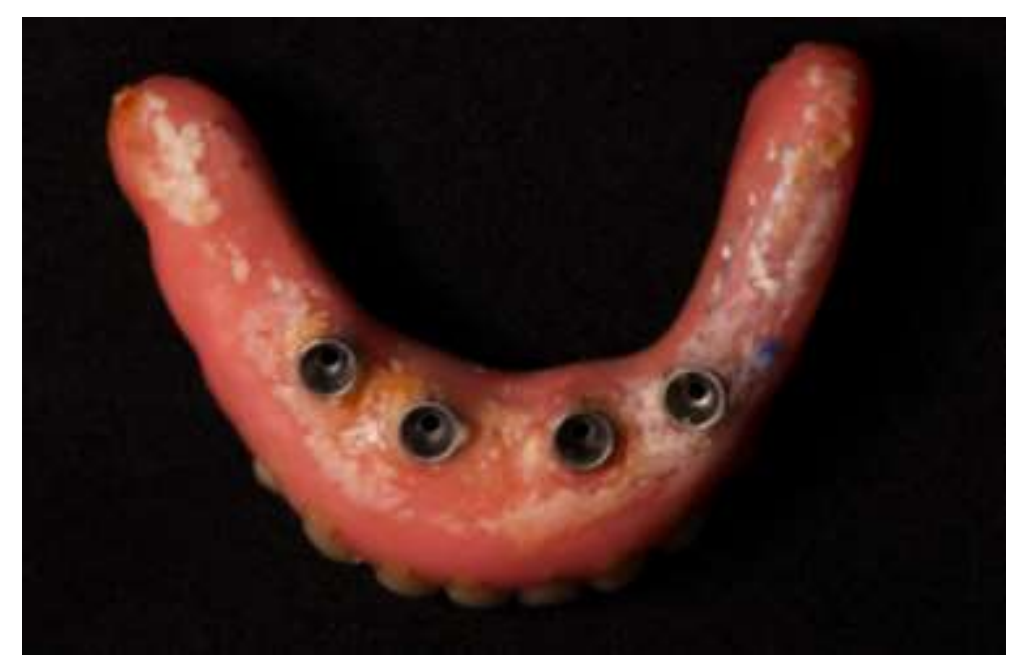

Fonte: Autores.

Após a remoção das próteses, procedeu-se a limpeza das mesmas e dos mini-pilares dos implantes. Em seguida foram realizadas as moldagens anatômicas superior e inferior com hidrocolóide irreversível, confecção dos modelos anatômicos com gesso pedra do tipo III e confecção das duas moldeiras individuais com resina acrílica auto polimerizável. Na sequência foram realizadas as moldagens funcionais com pasta a base de zinco e eugenol (superior) e com silicone de condensação (inferior). A moldagem funcional inferior foi realizada com a captura da posição dos implantes, para posteriormente o modelo de trabalho ser levado ao delineador para a avaliação da posição, inclinação e paralelismo entre os implantes (Figura 4). Os modelos de trabalho foram então confeccionados em gesso pedra tipo IV.

Figura 4: Modelo de trabalho inferior posicionado no delineador para verificação da posição, inclinação e paralelismo entre os implantes.

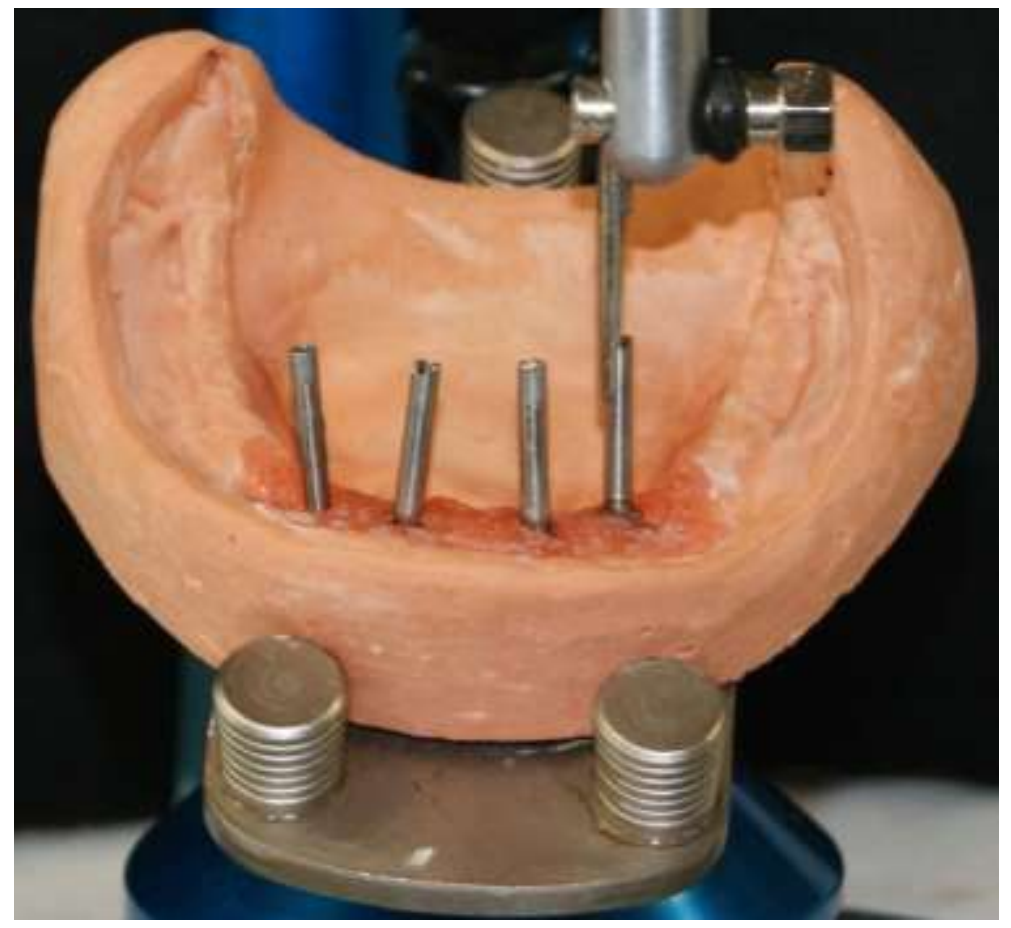

Fonte: Autores. 
Após a avaliação no delineador, foi possível planejar o sistema de retenção a ser utilizado no caso. O sistema de escolha foi o sistema independente Equator (Neodent, Curitiba, Brasil). Em seguida foram confeccionadas as bases de prova e planos de cera para realização do registro maxilo mandibular. Assim sendo, foram feitas todos as individualizações nos planos de cera (suporte de lábio, altura incisal, inclinação do plano oclusal, corredor bucal, linhas de referência, dimensão vertical de oclusão) e o registro em relação cêntrica. Na mesma sessão foi realizada a seleção da cor, forma e tamanho dos dentes artificiais.

Os modelos foram montados em articulador semi-ajustável e procedeu-se à montagem dos dentes artificiais selecionados. Após a montagem dos dentes, foi realizado o enceramento caracterizado com as ceras do sistema Tomas Gomes (STG) e as ceras gingiwax (Formaden, São Paulo, Brasil), para oferecer uma semelhança com a gengiva da paciente (Figura 5) e garantir maior satisfação e confiabilidade na prova funcional e estética dos dentes (Figura 6).

Figura 5: Montagem de dentes com enceramento caracterizado.

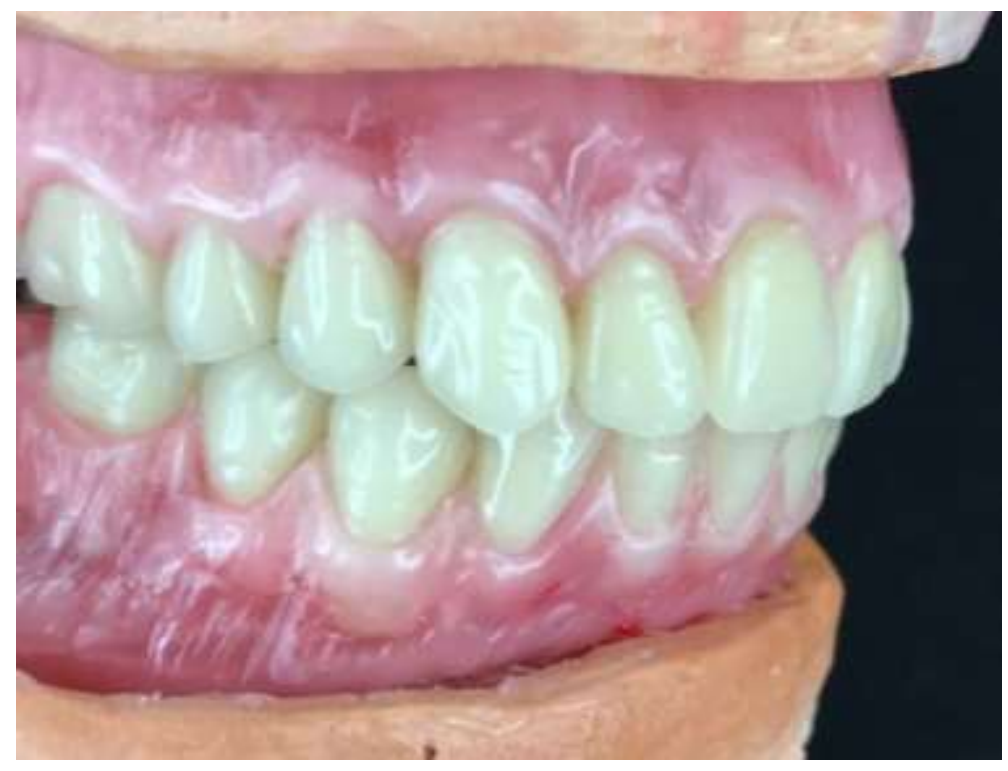

Fonte: Autores.

Figura 6: Prova funcional e estética dos dentes.

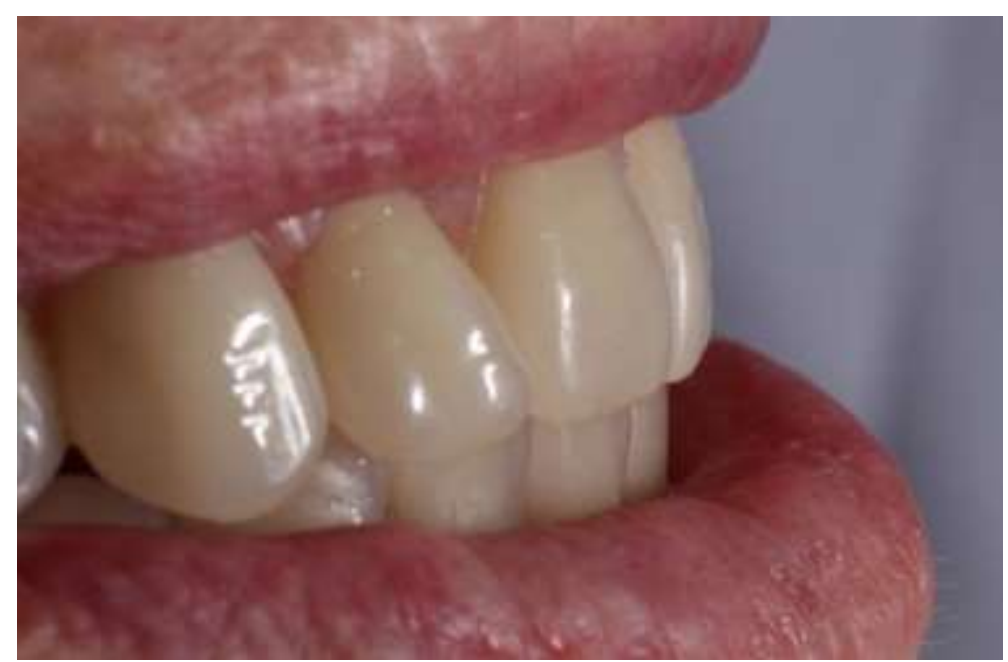

Fonte: Autores. 
A prova funcional e estética dos dentes foi realizada por meio de avaliação do suporte labial, altura incisal, inclinação do plano oclusal, tamanho corredor bucal, linha média, tamanho do incisivo central em relação à linha alta do sorriso, distância dos caninos, dimensão vertical de oclusão e espaço funcional livre e oclusão em relação cêntrica. Além disso, foram realizados fotografias e vídeos da paciente sorrindo e conversando para que a mesma pudesse ver como ficaria o resultado final das próteses e pudesse construir uma imagem de como os outros a veriam. Após a avaliação de todos os critérios supracitados e aprovação da paciente, as próteses enceradas foram levadas ao laboratório para o processamento de suas bases.

O processamento das bases compreendeu: remoção dos modelos do ASA, inclusão em mufla, lavagem da cera, caracterização em resina com o STG (número 4), polimerização por energia de microondas, desinclusão, acabamento e polimento. Como resultado, próteses totais convencionais bem polidas e naturais foram obtidas (Figura 7).

Figura 7: próteses finalizadas.

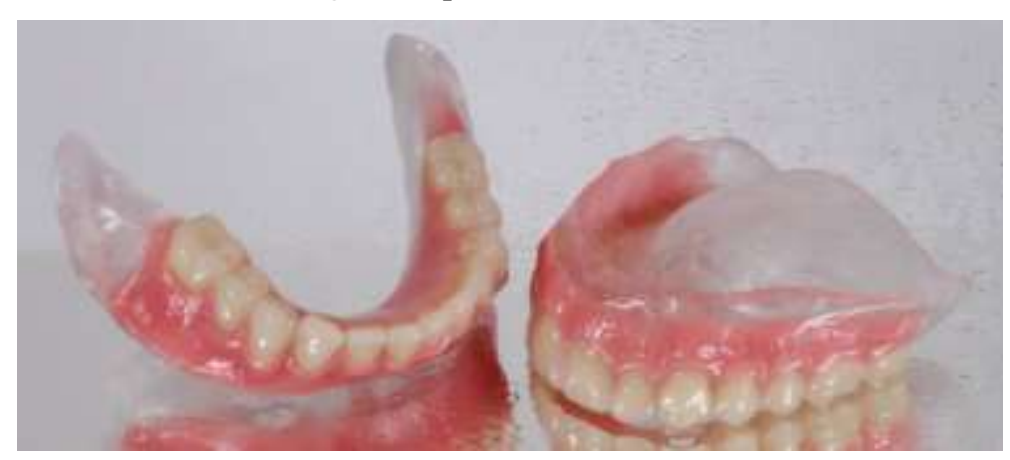

Fonte: Autores.

No momento da instalação das próteses, os pilares da prótese fixa tipo protocolo (mini-pilares) foram substituídos pelos pilares do sistema Equator (Figura 8 e 9) para que a captura da prótese pudesse ser executada. Foram realizados quatro desgastes na região interna da prótese correspondentes ao local onde seriam alojadas as cápsulas metálicas do sistema. Inicialmente somente duas cápsulas foram posicionadas sobre os pilares mais distais e procedeu-se a captura destas. Resina acrílica ativada quimicamente foi aplicada sobre as cápsulas e no alívio correspondentes destas na prótese. A prótese foi levada em boca, e solicitou-se que a paciente permanecesse em oclusão até a presa da resina. Em seguida, realizou-se o mesmo procedimento para os outros dois pilares. Após a polimerização da resina acrílica ativada quimicamente, retirou-se a prótese da boca da paciente e procedeu-se o preenchimento de eventuais espaços vazios entre as cápsulas e a base da prótese (Figura 10). 
Figura 8: Sistema equator. A) Pilar que será parafusado ao implante; B) Cápsulas metálicas com borrachas de nylon no seu interior que ficarão retidas na base da prótese.

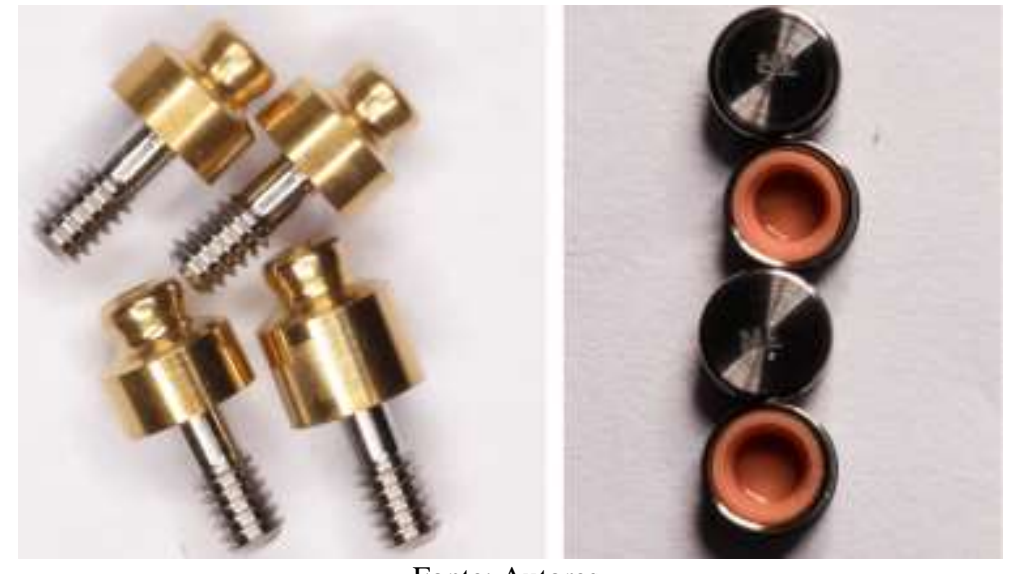

Fonte: Autores.

Figura 9: Substituição dos mini-pilares cônicos pelos pilares Equator. Pilares Equator posicionado nos dois implantes mais distais e vista oclusal do hexágono externo dos implantes centrais.

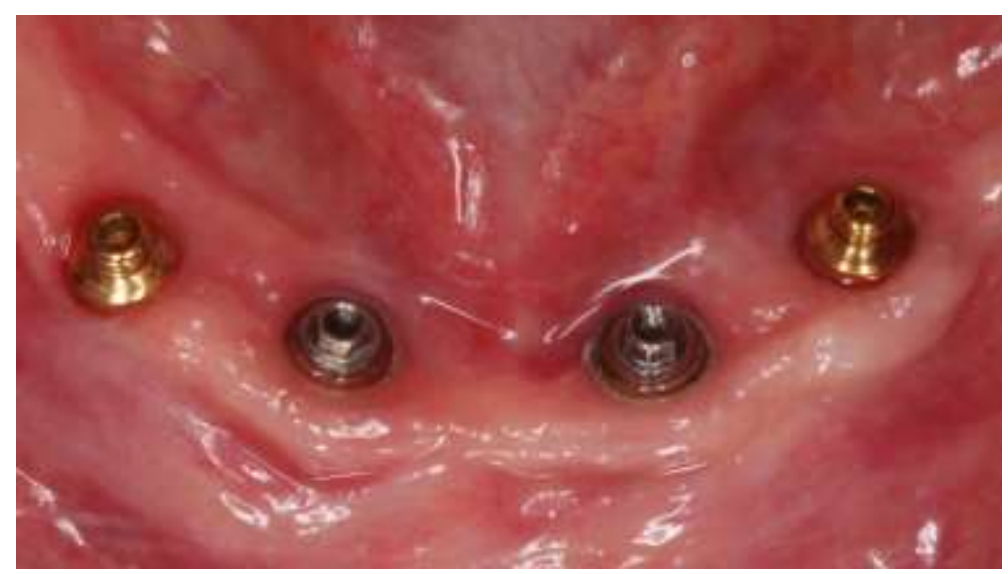

Fonte: Autores.

Figura 10: Finalização da captura com resina acrílica autopolimerizável das cápsulas metálicas do sistema de retenção com o preenchimento de eventuais espaços vazios entre as cápsulas e a base da prótese.

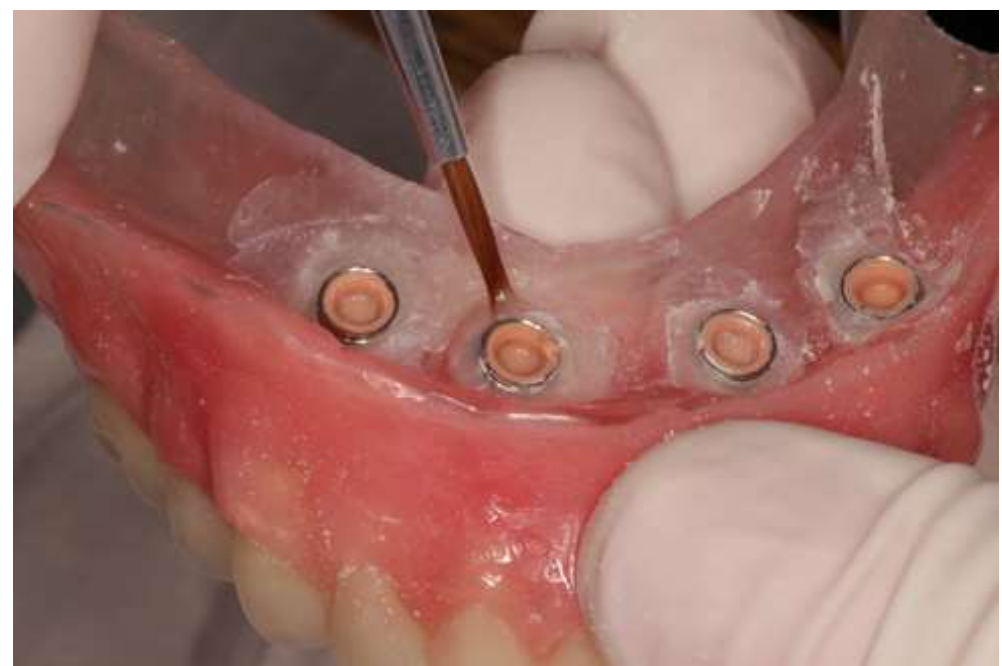

Fonte: Autores. 
Uma vez capturada a overdenture inferior e instalada a prótese total superior, a oclusão da paciente foi ajustada e foram fornecidas instruções para manutenção e higienização das próteses. Ao final, a paciente descreveu grande satisfação com as próteses (Figura 11) e com a capacidade de removê-las para higienização, de forma que o mau hálito foi extinto, possibilitando um convívio social despreocupado e prazeroso. Houve também relato de grande conforto ao mastigar e melhora da sintomatologia dolorosa da gengiva.

Figura 11: Aspecto final das próteses instaladas, devolvendo estética e função.

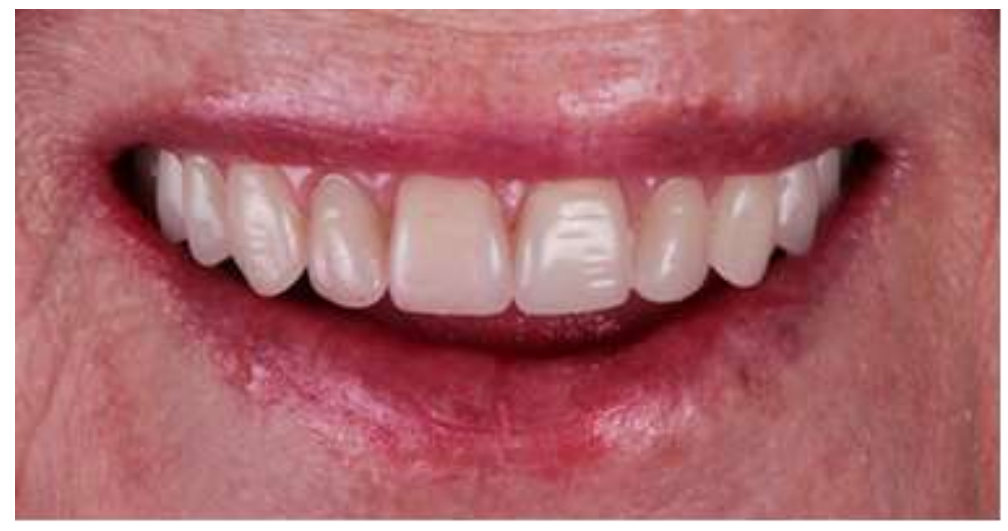

Fonte: Autores.

\section{Discussão}

Este caso se mostrou uma opção de tratamento eficiente ao substituir uma prótese total convencional superior e um protocolo metaloplástica inferior antigos e insatisfatórios por uma prótese total convencional superior e uma overdenture inferior novas. A prótese overdenture em comparação com a prótese total convencional apresenta maior retenção e estabilidade, o que favorece uma melhor eficiência mastigatória e maior força de mordida e consequentemente maior confiança no seu uso por parte do paciente (Sharma, Nagrath, \& Lahori, 2018). Além disso, apresenta uma facilidade de higienização quando comparada com a protocolo (de Almeida et al., 2015). Assim sendo a paciente que já estava acostumada com uma prótese inferior fixa não sentiu os possíveis efeitos de instabilidade de uma reabilitação removível convencional e sentiu-se mais confortável por poder removê-las da cavidade oral e poder realizar a higienização das próteses corretamente.

Dentro deste contexto, é preciso discutir o envelhecimento da população, somado ao aumento da expectativa de vida com saúde e o edentulismo. Estima-se que até 2040, o percentual de arcadas edêntulas será de 85,96\% entre idosos (Cardoso, Balducci, Telles, Lourenço, \& Nogueira, 2016). Isso significa que as altas taxas de edentulismo somadas ao aumento da expectativa de vida, suscita a discussão acerca dos planejamentos das reabilitações totais. É preciso avaliar não só as condições físicas e sistêmicas do paciente no momento da instalação dos implantes, mas também, como serão as condições de uso desses aparelhos ao longo dos anos. Ou seja, deve-se considerar o processo de envelhecimento, incluindo fenômenos biológicos, como a coordenação motora diminuída, que poderia afetar a higiene (Cooper et al.,2012). Um acúmulo de biofilme dental pode levar a quadros de peri-implantite, inflamação dos tecidos peri-implantares, exibindo clinicamente sangramento à sondagem, eventual supuração e hiperplasia da gengiva. Que quando não tratada, pode levar a extensa perda óssea e consequentemente perda do implante (de Oliveira et al., 2015).

No planejamento, também deve-se levar em conta que as próteses plásticas ou metaloplástica requerem sua substituição em um espaço de tempo menor que materiais como as cerâmicas. Embora os implantes e componentes protéticos tenham uma longevidade extensa, a resina dos dentes artificias e bases sofrem desgaste e degradação com o passar dos anos. O uso do mesmo aparelho por longo período pode gerar instabilidade oclusal, pelo desgaste das faces oclusais dos dentes 
artificiais, acúmulo de resíduos que incorporados à resina geram um odor desagradável, irritação e desconforto na mucosa oral (Del Bel Cury, Rodrigues Junior, \& Panzeri, 1996; Anusavice, 2013). Manifestações estas queixadas por parte da paciente do relato de caso.

Problemas com a saúde bucal podem interferir nos aspectos social e funcional dos pacientes, afetando não só a nutrição, fala, mas também o bem-estar e a autoestima (Fueki etal., 2007, Geckili et al., 2012). A reabilitação bem-sucedida de pacientes desdentados está relacionada ao conforto, função e estética, dessa forma, quando esses fatores são somados com um bem estar psicossocial, verifica-se um aumento da segurança, qualidade de vida e autoestima, causando efeitos positivos nas diversas esferas da sua vida (Acham et al, 2017; Kutkut et al., 2018; Gonçalves et al., 2020). Essa melhora pôde ser relatada pela própria paciente após a instalação e controle dos novos aparelhos.

No que tange ao planejamento do sistema de retenção da overdenture inferior, três possibilidades foram discutidas, visto que o espaço protético era de aproximadamente $14 \mathrm{~mm}$ o que permitia a colocação de diferentes sistemas de retenção (Bhargava et al., 2016). Essas possibilidades giraram em torno do uso de sistema barra clipe nos dois implantes anteriores e inutilização dos dois implantes posteriores; uso de sistema barra clipe nos dois implantes anteriores e sistema independente nos implantes posteriores ou o uso de sistema independente nos quatro implantes. Por apresentar facilidade técnica de execução, satisfatória relação custo benefício e uma facilidade maior de limpeza dos pilares e da prótese (Bhargava et al., 2016), optou-se pelo uso de um sistema independente.

Estão disponíveis no mercado uma variedade de sistemas independentes, dentre eles o sistema Equator, eleito para esse caso clínico. A escolha foi feita após o modelo de trabalho ser levado ao delineador e verificar que os implantes embora estivessem bem posicionados, não estavam paralelos entre si, e o fabricante indicar esses pilares para implantes com até $30^{\circ}$ de discrepância entre si. Isso é possível devido a redução da altura da parte ativa do pilar, permitindo a colocação da cápsula de captura em diferentes posições, otimizando o eixo de inserção da prótese (Elsyad et al., 2017; Bhargava et al., 2016; Macêdo et al., 2021). Acham et al. (2017) em seu estudo validou o presente planejamento ao utilizar também quatro implantes e esse mesmo sistema de retenção.

Assim sendo, dentro das limitações desse caso, foi dada uma solução eficaz e satisfatória para a paciente, garantindo função, estética e bem estar psíquico. Bem como, sanou a sua principal queixa que era a dificuldade de higienização do aparelho inferior.

\section{Conclusão}

O planejamento proposto e o tratamento executado foram eficazes e satisfatórios para a paciente. A confecção de uma nova prótese total convencional superior e uma overdenture inferior retida por sistemas independentes garantiu bem estar físico, psíquico e social e permitiu que a paciente pudesse realizar a higienização dos seus aparelhos protéticos de forma eficaz.

\section{Agradecimentos}

Ao programa de Integração Graduação/Curso Técnico em Prótese Dentária na Confecção de Próteses Totais e Overdentures para Pacientes Geriátricos da Faculdade de Odontologia da Universidade Federal de Uberlândia (FOUFU), curso Técnico em Prótese Dentária da Escola Técnica de Saúde (ESTES), Programa de Bolsas de Graduação (PBG) da Pró-reitoria de Graduação e a Faculdade de Odontologia da Universidade Federal de Uberlândia (FOUFU). 


\section{Referências}

Acham, S., Rugani, P., Truschnegg, A., Wildburger, A., Wegscheider, W. A., \& Jakse, N. (2017). Immediate loading of four interforaminal implants supporting a locator-retained mandibular overdenture in the elderly. Results of a 3-year randomized, controlled, prospective clinical study. Clin Implant Dent Relat Res, 19(5), 895-900. https://doi.org/10.1111/cid.12513

Adell, R., Eriksson, B., Lekholm, U., Brånemark, P. I., \& Jemt, T. (1990). Long-term follow-up study of osseointegrated implants in the treatment of totally edentulous jaws. The International journal of oral \& maxillofacial implants, 5(4), 347-359.

Alfadda, S. A., \& Attard, N. J. (2017). A cost analysis of a long-term prospective study of patients treated with immediately loaded implant-supported mandibular overdentures. Clin Implant Dent Relat Res, 19(5), 944-951. https://doi.org/10.1111/cid.12519

Anusavice, K. J. (2013). Phillips materiais dentários (12a ed.) Rio de Janeiro: Elsevier.

Babu, P. J., Alla, R. K., Alluri, V. R., Datla, S. R., \& Konakanchi, A. (2015). Dental Ceramics: Part I - An Overview of Composition, Structure and Properties. American Journal of Materials Engineering and Technology, 3(1), 13-18. DOI: 10.12691/materials-3-1-3

Bhargava, A., Sehgal, M., Gupta, S., \& Mehra, P. (2016). Classification system on the selection of number of implants and superstructure design on the basis available vertical restorative space and interforaminal distance for implant supported mandibular overdenture. J Indian Prosthodont Soc, 16(2), 131-135. https://doi.org/10.4103/0972-4052.176541

Cardoso, M., Balducci, I., Telles, D. M., Lourenço, E. J. V., \& Nogueira, L. Jr. (2016). Edentulism in Brazil: trends, projections and expectations until 2040. Ciênc Saúde Coletiva, 21(4):1239-45. https://doi.org/10.1590/1413-81232015214.13672015

Chung, K. H., Chung, C. Y., Cagna, D. R., \& Cronin, R. J., Jr. (2004). Retention characteristics of attachment systems for implant overdentures. J Prosthodont, 13(4), 221-226. https://doi.org/10.1111/j.1532-849X.2004.04042.x

Cooper, L. F., Limmer, B. M., \& Gates, W. D. (2012). "Rules of 10"--guidelines for successful planning and treatment of mandibular edentulism using dental implants. Compend Contin Educ Dent, 33(5), 328-334; quiz 335-326. http://www.ncbi.nlm.nih.gov/pubmed/22616215

Cordioli, G., Majzoub, Z., \& Castagna, S. (1997). Mandibular overdentures anchored to single implants: a five-year prospective study. J Prosthet Dent, 78(2), 159-165. https://doi.org/10.1016/s0022-3913(97)70120-3

da Silva, E.T., de Oliveira, R. T., \& Leles, C. R. (2015). O edentulismo no Brasil: epidemiologia, rede assistencial e produção de próteses pelo Sistema Único de Saúde. Tempus: actas de saúde coletiva, 9(3), 121-134. https://doi.org/10.18569/tempus.v9i3.1790

Datla, S. R., Alla, R. K., Alluri, V. R., P, J. B., \& Konakanchi, A. (2015). Dental Ceramics: Part II - Recent Advances in Dental Ceramics. American Journal of Materials Engineering and Technology, 3(2), 19-26. https://doi.org/10.12691/materials-3-2-1.

de Almeida, H. C. R., Santana, E. T. S. de, Santos, N. A. T. dos, Moraes, P. K. M. de, Araújo, Y. R. F. de, \& Gerbi, M. E. M. de M. (2015). Clinical aspects in the treatment planning for rehabilitation with overdenture and protocol-type prosthesis. RGO - Revista Gaúcha de Odontologia, 63(3), 271-276. https://doi.org/10.1590/1981-863720150003000032920

de Freitas, D. F., Freire, J. C. P., Melo, W. O. de S.., Figueirêdo Júnior, E. C., Costa, B. P., Formiga Filho, A. L. N., Lima, S. A. N. de., Pereira, J. V., \& Ribeiro, E. D. (2020). All-on-four treatment in implantology: concept and updates. Research, Society and Development, 9(10), e4079106045. https://doi.org/10.33448/rsd-v9i10.6045

Del Bel Cury, A. A., Rodrigues Junior, A. L., \& Panzeri, H. (1994). Resinas acrílicas dentais polimerizadas por energia de microondas, método convencional de banho de água e quimicamente ativada: propriedades físicas. Revista de Odontologia da Universidade de São Paulo, 8(4), $243-249$.

Delwel, S., Binnekade, T. T., Perez, R., Hertogh, C., Scherder, E. J. A., \& Lobbezoo, F. (2018). Oral hygiene and oral health in older people with dementia: a comprehensive review with focus on oral soft tissues. Clin Oral Investig, 22(1), 93-108. https://doi.org/10.1007/s00784-017-2264-2

de Oliveira, M. C., Corrêa, D. F. M., Laurêdo, L. F. B., de Mendonça, L. P. F., de Lemos, A. B., do Carmo, \& G. G. W. (2015). Peri-implantite: etiologia e tratamento. Revista Brasileira de Odontologia, 72 (1-2), 96-99.

de Souza, F. I., de Souza Costa, A., dos Santos Pereira, R., dos Santos, P. H., de Brito, R. B., Jr., \& Rocha, E. P. (2016). Assessment of Satisfaction Level of Edentulous Patients Rehabilitated with Implant-Supported Prostheses. Int J Oral Maxillofac Implants, 31(4), 884-890. https://doi.org/10.11607/jomi.4267

Elsyad, M. A., \& Khairallah, A. S. (2017). Chewing efficiency and maximum bite force with different attachment systems of implant overdentures: a crossover study. Clin Oral Implants Res, 28(6), 677-682. https://doi.org/10.1111/clr.12861

Elsyad, M. A., \& Shawky, A. F. (2017). Masticatory function with ball and resilient telescopic anchors of mandibular implant-retained overdentures: A crossover study. Quintessence Int, 48(8), 615-623. https://doi.org/10.3290/j.qi.a38555

Fueki, K., Kimoto, K., Ogawa, T., \& Garrett, N. R. (2007). Effect of implant-supported or retained dentures on masticatory performance: a systematic review. J Prosthet Dent, 98(6), 470-477. https://doi.org/10.1016/S0022-3913(07)60147-4

Geckili, O., Bilhan, H., Mumcu, E., Dayan, C., Yabul, A., \& Tuncer, N. (2012). Comparison of patient satisfaction, quality of life, and bite force between elderly edentulous patients wearing mandibular two implant-supported overdentures and conventional complete dentures after 4 years. Spec Care Dentist, 32(4), 136-141. https://doi.org/10.1111/j.1754-4505.2012.00258.x

Gonçalves, F. P., Alves, G., Oliveira, F., Antunes, L. A. A., Soares, J. R. A., Perazzo, M. F., Paiva, S. M., \& Scelza, M. F. Z. (2020). Impacto da reabilitação oral na qualidade de vida e nos níveis de cortisol de pacientes geriátricos. Research, Society and Development, 9(11), e2639119911. https://doi.org/10.33448/rsd-v9i11.9911 
Research, Society and Development, v. 10, n. 2, e26710212431, 2021

(CC BY 4.0) | ISSN 2525-3409 | DOI: http://dx.doi.org/10.33448/rsd-v10i2.12431

Kern, J. S., Kern, T., Wolfart, S., \& Heussen, N. (2016). A systematic review and meta-analysis of removable and fixed implant-supported prostheses in edentulous jaws: post-loading implant loss. Clin Oral Implants Res, 27(2), 174-195. https://doi.org/10.1111/clr.12531

Kutkut, A., Bertoli, E., Frazer, R., Pinto-Sinai, G., Fuentealba Hidalgo, R., \& Studts, J. (2018). A systematic review of studies comparing conventional complete denture and implant retained overdenture. J Prosthodont Res, 62(1), 1-9. https://doi.org/10.1016/j.jpor.2017.06.004

Macêdo, T. C. S., Lopes, C. de C. A., Gonçalves, F. S., Cerda-Rizo, E. R., Mancia, B. L. P., \& Silverio, M. G. de C. (2021). Replacement of bar-clip retention system by Equator in overdenture on implant. Research, Society and Development, 10(1), e38710111829. https://doi.org/10.33448/rsd-v10i1.11829

Naert, I., Quirynen, M., Hooghe, M., \& van Steenberghe, D. (1994). A comparative prospective study of splinted and unsplinted Branemark implants in mandibular overdenture therapy: A preliminary report. The Journal Of Prosthetic Dentistry, 71(5), 486-492. http://dx.doi.org/10.1016/0022-3913(94)90188-0.

Pereira, A. S., Shitsuka, D. M., Parreira, F. J., \& Shitsuka, R. (2018). Metodologia do trabalho científico. Ed. UAB/NTE/UFSM. https://repositorio. ufsm. br/bitstream/handle/1/15824/Lic_Computacao_Metodologia-Pesquisa-Cientifica.pdf

Rivaldo, E. G., Montagner, A., Nary, H., da Fontoura Frasca, L. C., \& Branemark, P. I. (2012). Assessment of rehabilitation in edentulous patients treated with an immediately loaded complete fixed mandibular prosthesis supported by three implants. Int J Oral Maxillofac Implants, 27(3), 695-702. http://www.ncbi.nlm.nih.gov/pubmed/22616065

Sánchez-Siles, M., Ballester-Ferrandis, J. F., Salazar-Sánchez, N., Gómez-García, F. J., Moraleja-Ruiz, R., \& Camacho-Alonso, F. (2018). Long-term evaluation of quality of life and satisfaction between implant bar overdentures and conventional complete dentures: A 23 years retrospective study. Clinical implant dentistry and related research, 20(2), 208-214. https://doi.org/10.1111/cid.12576

Sharma, A. J., Nagrath, R., \& Lahori, M. (2017). A comparative evaluation of chewing efficiency, masticatory bite force, and patient satisfaction between conventional denture and implant-supported mandibular overdenture: An in vivo study. Journal of Indian Prosthodontic Society, 17(4), 361-372. https://doi.org/10.4103/jips.jips_76_17

Scherer, M. D., McGlumphy, E. A., Seghi, R. R., \& Campagni, W. V. (2014). Comparison of retention and stability of two implant-retained overdentures based on implant location. J Prosthet Dent, 112(3), 515-521. https://doi.org/10.1016/j.prosdent.2014.03.003

van der Bilt, A., van Kampen, F. M., \& Cune, M. S. (2006). Masticatory function with mandibular implant-supported overdentures fitted with different attachment types. Eur J Oral Sci, 114(3), 191-196. https://doi.org/10.1111/j.1600-0722.2006.00356.x

Zancope, K., Simamoto Junior, P. C., Davi, L. R., Prado, C. J., \& Neves, F. D. (2014). Immediate loading implants with mandibular overdenture: a 48 -month prospective follow-up study. Braz Oral Res, 28. https://doi.org/10.1590/1807-3107bor-2014.vol28.0030 